\section{Mosquito control}

The Inz-ECO solution

Mosquitoes are vectors for diseases, such as yellow fever, dengue, chikungunya and

Zika, which pose a threat to

human health on a global scale.

Mosquito control is therefore

an urgent consideration for

public health. Dr Philip Koenler

the University of Florida hav

pioneered two innovative

Inz-ECO mosquito chips and

Inz-ECO mosquito traps, which

can be used in combination

with education and other

control methods to massively

reduce mosquito population

and therefore help preve

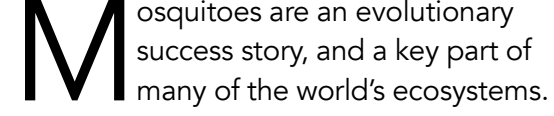
one of the deadliest animals on the planet. To some fortunate humans, mosquitoes are little more than a buzzing, biting annoyance. To others, numerous potentially deadly diseases.

Of the more than 3,000 species of mosquito, just three genera bear most of the responsibility for transmitting diseases to humans. Aedes mosquitoes carry, among other things, dengue and yellow fever; Culex are responsible for encephalitis and the West Nile virus. the most well-known mosquito-borne . insecticides poses a challenge. mosquitoes, A. aegypti usually feeds during the day. This means that many methods of mosquito control disease: malaria.

Mosquitoes are considered one of the world's biggest health threats, with children and elderly people in developing countries most at risk from mosquito-bourne disease. There is an urgent and ongoing need for new methods of mosquito control. This as, thanks to climate change, some mosquitoes are known to be expanding their geographical ranges, potentially putting millions more at risk.

\section{THE AEDES CHALLENGE}

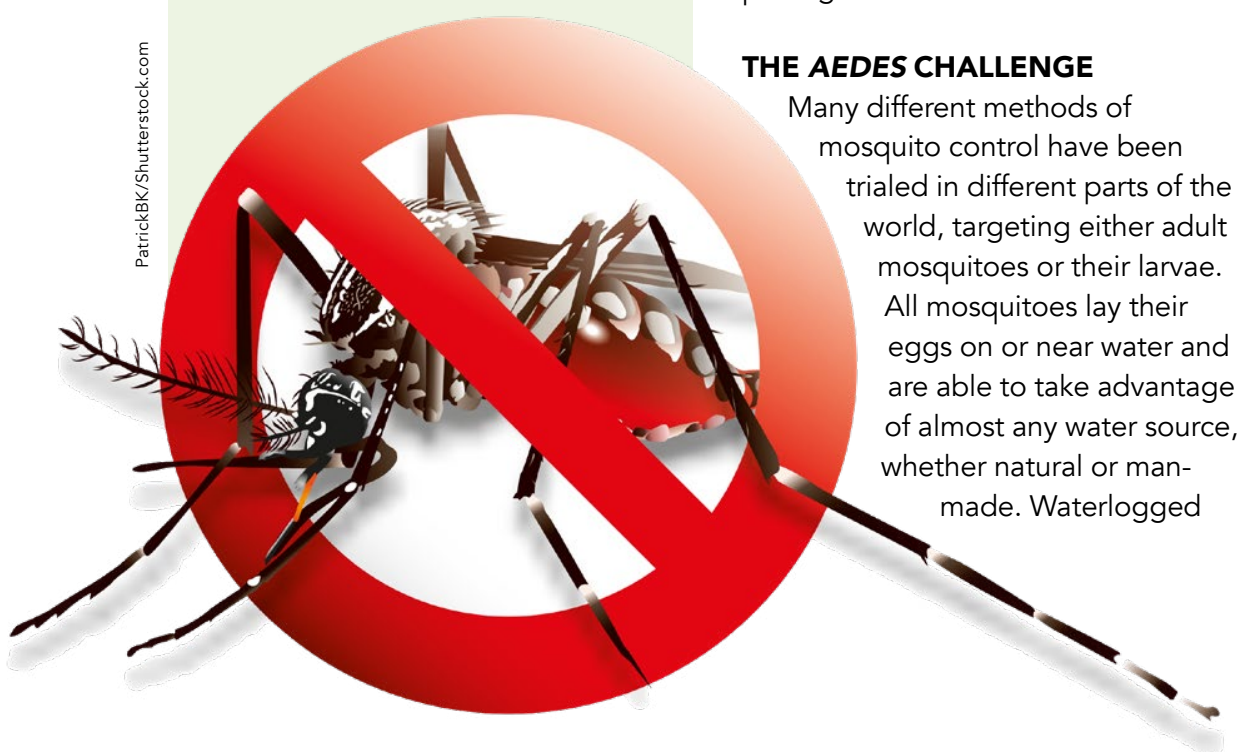

clogged gutters and bird bath delling larvae is therefore a potentially

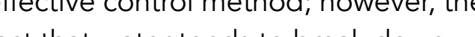

Controlling the Aedes agypinosquito Aedes aegyptimosquito is a particularly difficult problem. This fever mosquito - previously evolved in its native Africa. However, A aegypt thas expanded its range at an astonishing rate and is now found on every contine except Antarctica. Unlike most other common control measures, which focus on killing mosquitoes after sunset, are ineffective against $A$. aegypti. In addition, female $A$. aegypti demonstrate "skip oviposition;" basically, this means that they can lay eggs in several different water containers in a relatively short space of time. Skip oviposition means that the "source reduction" control method, in which water-holding emptied, is less likely to be effective.

\section{IMPROVING MOSOUITO CONTROL} Chemical contr added to water to kill the developing mosquito larvae, is promising, but tricky to apply in practice. The lavicides may come in the form of liquid, granules or pellets. In the past, some popula larvicides (for example, Abate) belonged to the class of chemicals called organophosphates. These chemicals, which cause death by disrupting the nervous system, are now banned in the Wilue to their harmful effects on other wildlife and potential danger to humans. Insect growth regulators, which interfere with larval development, are useful in
destroying mosquitoes at the larval

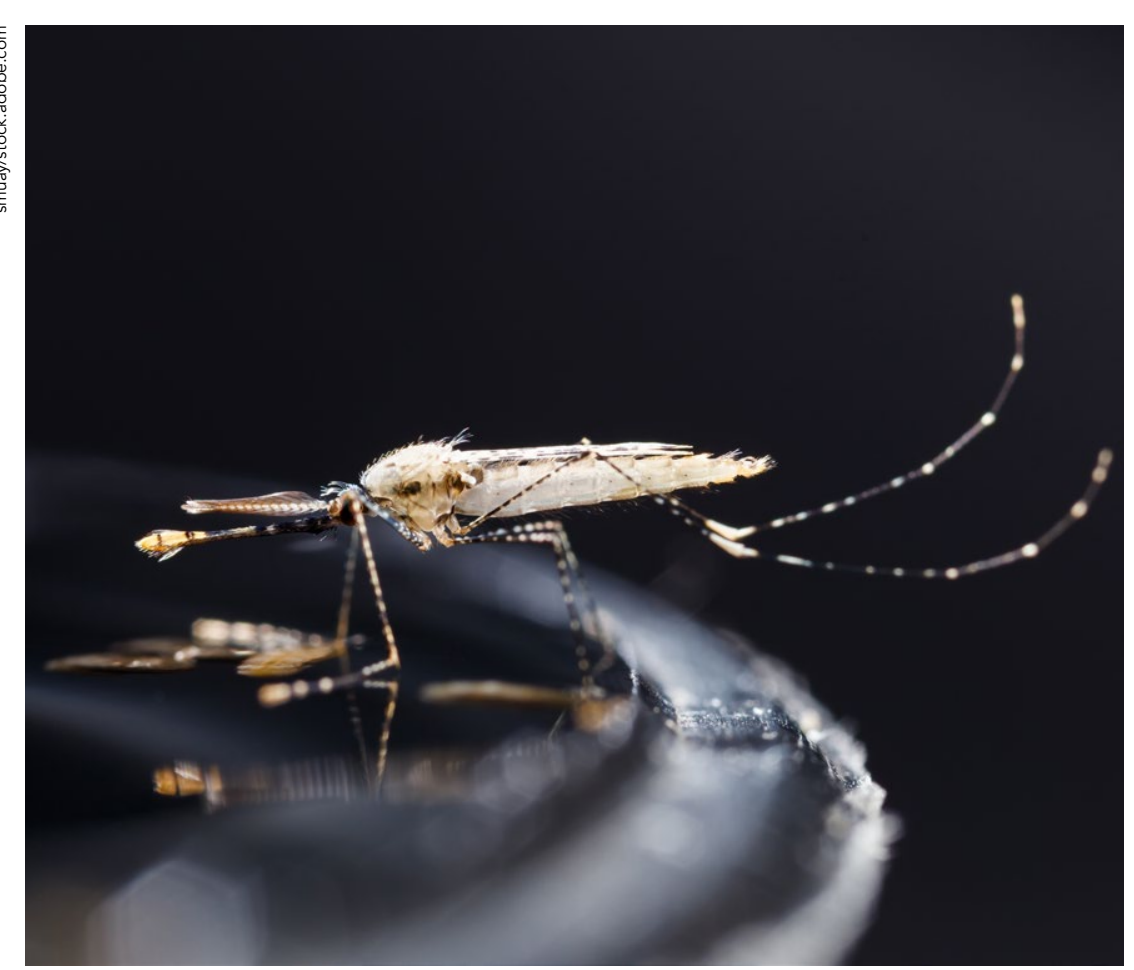

Ir-ECO's durable ovitraps exploit the mosquitoes' need to lay eggs in water-holding containers

In laboratory studies, the trap resulted in 95-100\% mortality of laying females and prevented all eggs from hatching.

or pupal stage. However, this type of mosquito control also has several drawbacks: when applied to water, it must be applied at the correct larval stage to be effective, it is relatively slow; and it only lasts as long as there is water the container.

To address some of the current problems with mosquito control - and Philip Koehler and Dr Roberto Pereir of the University of Florida teamed up with Enrico Levi of Inz-ECO. As a result of this collaboration, Inz-ECO have developed two innovative mosquito-control

products: an ovitrap to capture and kill female mosquitoes, to prevent egglaying and subsequent developmen of larvae; and mosquitocidal chips, which can be added to any container of standing water.

\section{NZ-ECO MOSQUITO TRAPS} Lethal ovitraps exploit the mosquitoes need to lay eggs in water-holding containers. The trap developed by InzECO is a dura, dual ofion ovitrap.

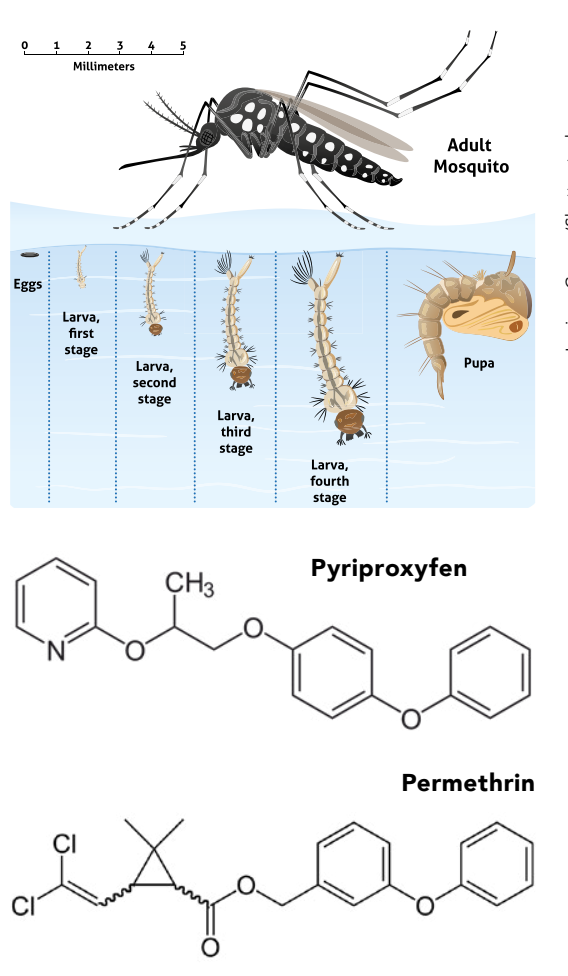

simple and quick to set up - users need only add water - and lasts for three months. Importantly, this control method is also safe for other animals, as the insecticide is contained within the trap.

Ovitraps like the Inz-ECO product may be the most effective method so far

for control of A. aegypti. In laboratory studies, the trap resulted in 95-100\% mortality of laying females and prevented all eggs from developing into biting adult mosquitoes. The traps recively prevented adult mosquito mertity in a ca med a test $50 \%$ six months.

INZ-ECO MOSOUITO CHIPS
While the Inz-ECO trap has many

advantages, a different option is needed

for existing water sources. For this

purpose, the team created Inz-ECO

chips. These mosquitocidal chips can

be added to small water sources or

containers, such as bird baths, garden

ponds or drains. A micro-dose of the

lanvicide pyriproxyfen is embedded

into the surface of the chips. In a similar formulation to that used in the traps, the chemical is contained within a polymer formulation to prevent degradation and allow slow release of the insecticide into the water. Each chip effectively treats up to 20 liters of water; for larger contain
more chips can simply be added. 
The chips, which are effective for three months, are intended to be added to the mosquito breding season. The chips are durable, remaining active even when containers are filled and re-filled more than 1,000 times. This method of mosquito control is environmentallyfriendly: according to the World Health Organization, pyriproxyfen is safe for us around pets, birds and other wildlife. According to WHO standards, potable water that has been treated with the chips remains safe to drink.

\section{SUPPORTING PUBLIC HEALTH}

Recently, the University of Florida collaborated with Operation Blessing, which provides services such as food clean water and disaster relief to mosquito-control program in Hond a

One particular suburb of the second largest city in Honduras, Monte Verde, in western Honduras, was in desperate need of new mosquito-control solutions. In this area, there is no piped water weekly water deliveries. After transpo the water is stored in various types of containers, including drums and buckets. Residents also dig wells that are lined with tires. Unfortunately, this type of water storage creates an ide environment for mosquitoes to lay their eggs. As a result, the town was experiencing frequent outbreaks of virus and Zika disease.
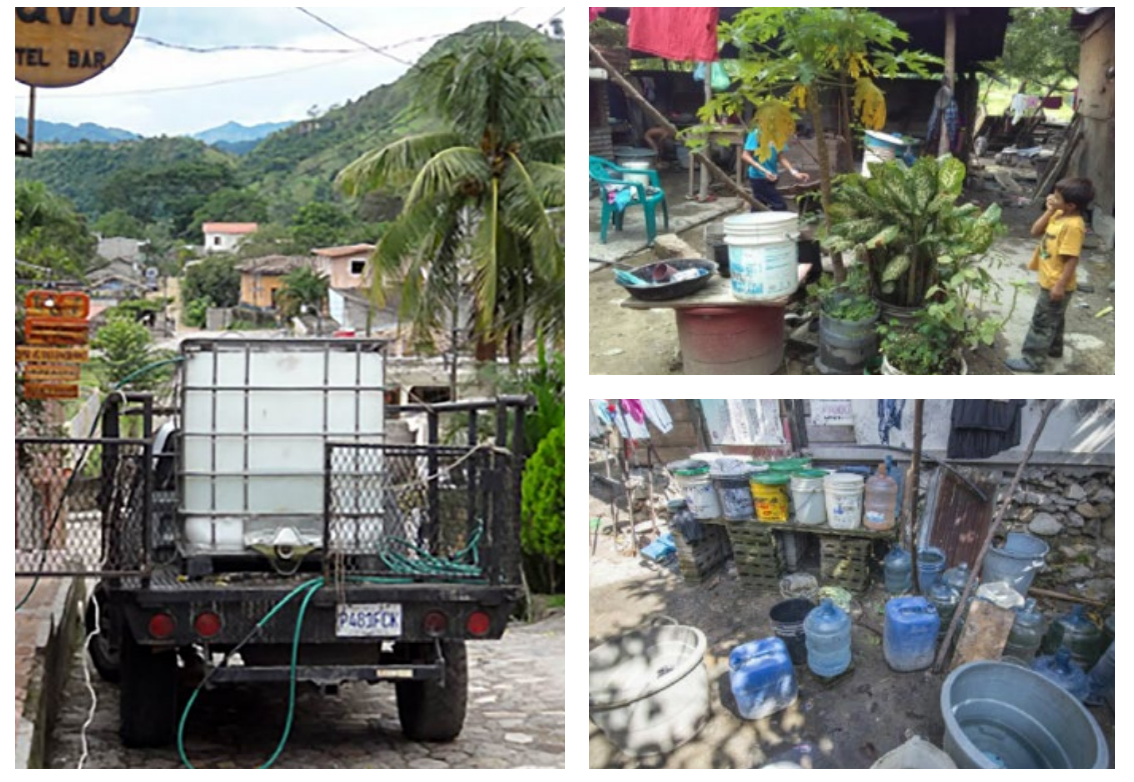

The chips are durable, remaining active even when containers are filled and re-filled more than 1,000 times.

program of mosquito control. A key part the container. Rather than preventing of the project was the use of Inz-ECO mosquitoes from laying their eggs in chips in all appropriate water containers. water containers, the stickers destroyed Alongside this, local school children were the developing larvae, allowing for educated in mosquito management. Fish, turtles and crustaceans were placed in wells to feed on the mosquito larvae, in a form of biological control.

The program also trialed another innovative Inz-ECO produc mosqui cidal stickers. These stickers, stickers can be applipd to thoyfen. The water containers, so that they stay in place even when water is dumped from

Together with Operation Blessing, the

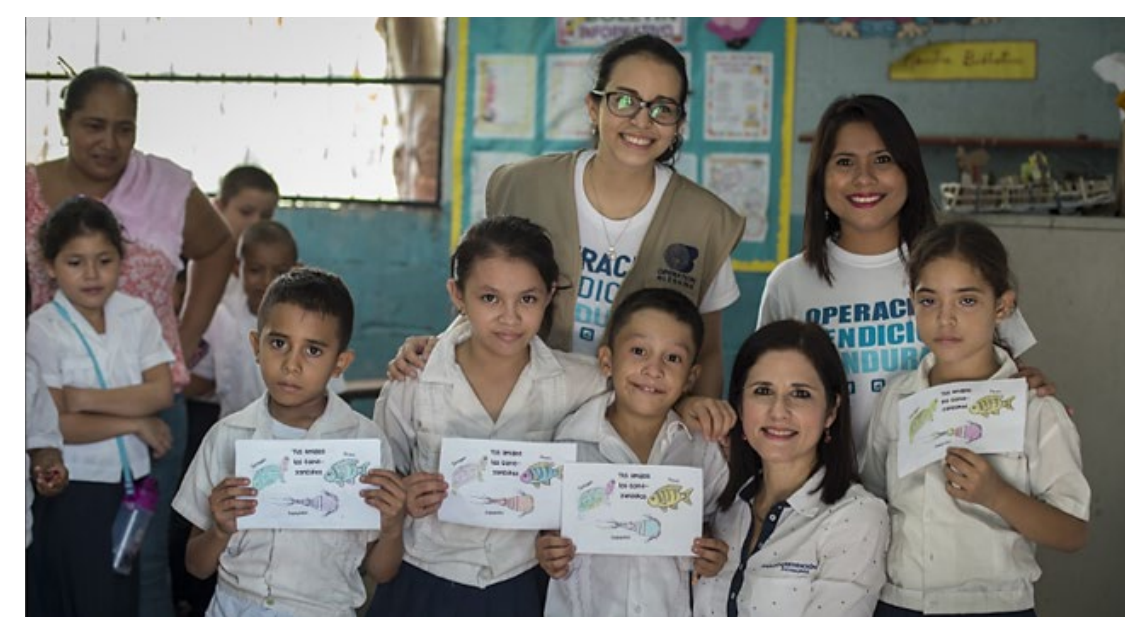

Educational program in Monte Verde, Honduras, in collaboration with Operation Blessing.
The project was hugely successful. This was seen not only in a much-reduced mosquito population, but also in protection from cases of mosquitoborne diseases. In Choloma, a region carrounding Monte Verde, there were 71 cases of dengue, three of Zika and one Monte Verde, there were none.

In Monte Verde, the results were clear: a combination of Inz-ECO chips and stickers with education and biologica control reduced mosquito numbers to a level where the local population was protected. The traps, chips and stickers developed by Dr Koehler and Pereira and Inz-ECO show huge promise for The products will soun be available targeted - and will be an important too in the ongoing attempts to control the world's expanding mosquito population. Find out more at https://www.inz-eco.com/ effective population control. for purchase - a spring 2021 release is

\section{Behind the Research}
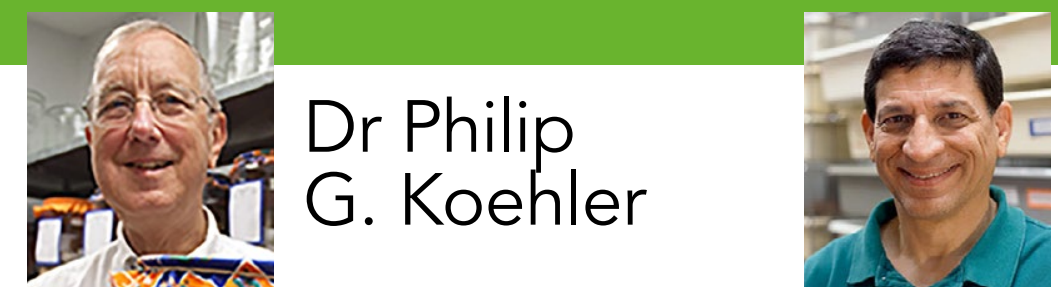

Dr Roberto

M. Pereira

E: randi@inz-eco.com T: +1 (844) 944-2601 W: $\mathbf{h t t p s : / / w w w . i n z - e c o . c o m / ~}$
@@inz_eco

Research Objectives

Inz-ECO provides next-generation, award-winning effective and eco-friendly pest control products.

\section{Detail}

\section{Bio}

Dr Philip G. Koehler is Professo Emeritus in Urban Entomology at has been awarded as Fellow of the National Academy of Inventors and Pest Control Hall of Fame.
Dr Roberto M. Pereira, is a Research Scientist of Urban Entomology at University of Florida. Dr Pereira conducting research and extension on different aspects of the biology mosquitoes and other insects that live in and around human structures.
Funding

Dr Philip Koehler, Dr Roberto Pereira, Conse

Collaborators

Dr Philip Koehler, Dr Roberto Pereira, Enrico Paolo Levi, Casey Parker, and Kristen Coleen Stevens

\section{References}

Parker C. (2016) Surveillance and control of Aedes aegypti and Aedes albopictus with a novel lethal ovitrap. Thesis presented to the University of Florida.

Parker C, Pereira R, Baldwin R, Chashopoulou A, Koehler P. (2017) Laboratory Evaluation of a Novel Lethal Ovitrap for 54 (6), 1666-1673. doi: 10.1093/jme/tix161.

Stevens K. (2017) Control of Aedes aegypti using mosquitocidal chips containing the insect growth regulator pyriproxyfen. Thesis presented to the University of Florid.

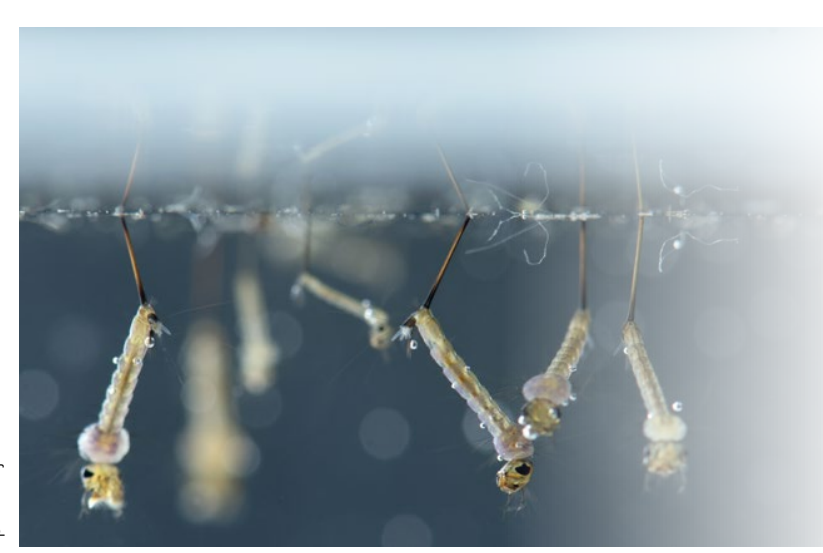

\section{Personal Response}

Are there similar eco-friendly pest control systems in the pipeline at Inz-ECO?

II We are working on industrializing a new set of fly traps and devices, both for professional and domestic use. Also these products are based on R\&D funded by
the Pentagon. In this case, the products will be more the Pentagon. In this case, the products will be more
effective and longer lasting than what is currently effective and longer last
available on the market.

We are also testing new products for cockroaches and bed bugs. Some are pesticide-free, others involve micro-use of pesticides in innovative ways.

SIMPLE | INNOVATIVE | PROTECTION 